\title{
Charged-Current Electron Neutrino Measurement with the MicroBooNE Detector
}

\section{Wouter Van De Pontseele ${ }^{a, b, *}$ for the MicroBooNE Collaboration}

${ }^{a}$ Department of Physics, University of Oxford,

Denys Wilkinson Building, Keble road, Oxford, UK

${ }^{b}$ Department of Physics, Harvard University,

18 Hammond St, Cambridge, MA, USA

E-mail: woutervdp@g.harvard.edu

MicroBooNE is the first phase of Fermilab's Short Baseline Neutrino (SBN) Liquid Argon Time Projection Chamber (LArTPC) programme [1]. This proceeding outlays the first characterisation of electron neutrinos in a muon neutrino beam with the LArTPC detector technology. The Booster Neutrino Beam has an energy peaking around $1 \mathrm{GeV}$ and an electron content of approximately $0.5 \%$ [2]. The analysis investigates electrons produced in charged-current electron neutrino interactions. The kinematics of the electrons are measured along with comparisons to simulation. Most of the systematic uncertainties are constrained using a data-driven sample of charged-current muon neutrino events. The measurement of electron neutrinos originating from the Booster Neutrino Beam is a crucial component to understand the nature of the observed excess of low energy electromagnetic-like events at MiniBooNE [3].

40th International Conference on High Energy physics - ICHEP2020

July 28 - August 6, 2020

Prague, Czech Republic (virtual meeting)

\footnotetext{
${ }^{*}$ Speaker
} 


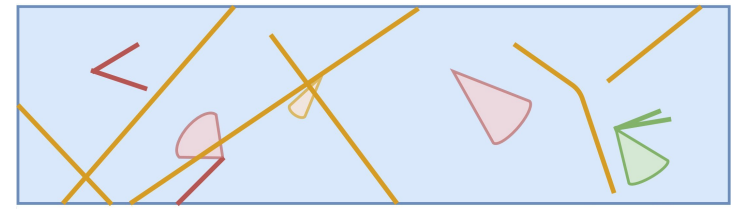

(a) Typical event with multiple interactions isolated by Pandora in cosmic muons (yellow) and neutrino-like slices (red and green).

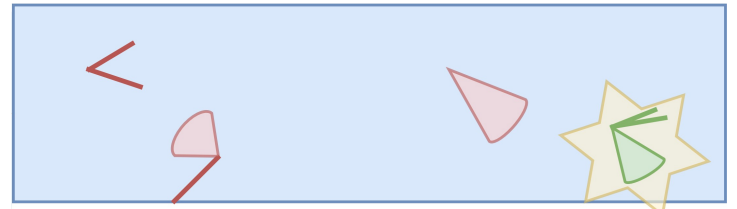

(b) Event after the removal of obvious cosmics tagged geometrically by Pandora. The neutrino slice (green) is selected using the light information.

Figure 1: Succession of steps in cosmic removal performed using Pandora's topological pattern recognition combined with scintillation light information. The lines and the cones represent track-like and electromagnetic shower particles respectively.

\section{Introduction}

Electron neutrino detection in MicroBooNE is made challenging by a variety of factors. First, the event readout in LArTPC technology requires advanced noise handling and signal processing techniques $[4,5]$. These signals are then reconstructed into particle objects using the Pandora event reconstruction framework [6]. Most of the collected charge in the TPC originates from cosmic activity (section 2). Once neutrino events are isolated from cosmic backgrounds, novel particle identification algorithms are used to identify electron showers and reject but muons from chargedcurrent $v_{\mu}$ interactions and photon showers from $\pi^{0}$ decay (section 3). These tools are combined into an inclusive charged current electron neutrino $\left(v_{e} \mathrm{CC}\right)$ selection in section 4 . This selection is developed on a sub-sample corresponding to $5 \times 10^{19}$ POT to preserve blindness in the low-energy region. Results containing high-energy events on an extensive $6 \times 10^{20}$ POT data-set are shown in section 5 .

\section{Cosmic Rejection}

MicroBooNE is located in an open pit approximately $6 \mathrm{~m}$ below ground level and subject to an atmospheric muon rate of $O(5 \mathrm{kHz})$. Prompt scintillation light in coincidence with the beam signal is used to select event containing a neutrino interaction [7]. As shown in fig. 1, these events are still dominated by cosmic charge deposits due to the $O(\mathrm{~ms})$ drift window. A set of tools is used to identify backgrounds: geometrically crossing the detector, containing a stopping muon Bragg peak, crossing the cathode or anode plane. Finally, the cluster of charge which is most compatible with the PMT signal is selected.

\section{Particle identification}

The selected cluster of charge deposits is reconstructed as a set of particles that are either track- or shower-like. To distinguish $v_{e} \mathrm{CC}$ interactions from other neutrino interactions, particle identification is crucial. 


\subsection{Proton-Muon Separation}

The lion share of tracks are either muons (from cosmic activity or $v_{\mu}$ interactions) or protons. These can be distinguished by using the ionisation profile as described by the Bethe-Bloch formula. For heavier protons, a shorter and more intense Bragg peak is expected. For muons, that are minimum ionising particle at the energies of interest, a lower ionisation is expected. The complex detector anisotropies complicate the picture, therefore, a likelihood ratio approach is used as test statistic $\mathcal{T}$. This likelihood is evaluated for each wire plane in function of the ionisation $(\mathrm{d} E / \mathrm{d} x)$, the residual range in the track (segment) and the angle with the wires:

$$
\mathcal{T}(\mathrm{d} E / \mathrm{d} x \text {, segment }, \theta)=\frac{\mathcal{L}(\text { muon } \mid \mathrm{d} E / \mathrm{d} x \text {, segment }, \theta)}{\mathcal{L}(\text { proton } \mid \mathrm{d} E / \mathrm{d} x, \text { segment }, \theta)} .
$$

The likelihood for the different planes can be multiplied into the combined liklihood:

$$
\mathcal{L}(U, V, Y)=\mathcal{L}(U) \times \mathcal{L}(V) \times \mathcal{L}(Y) .
$$

The performance of the likelihood ratio as track PID is shown in the right panel of fig. 2.
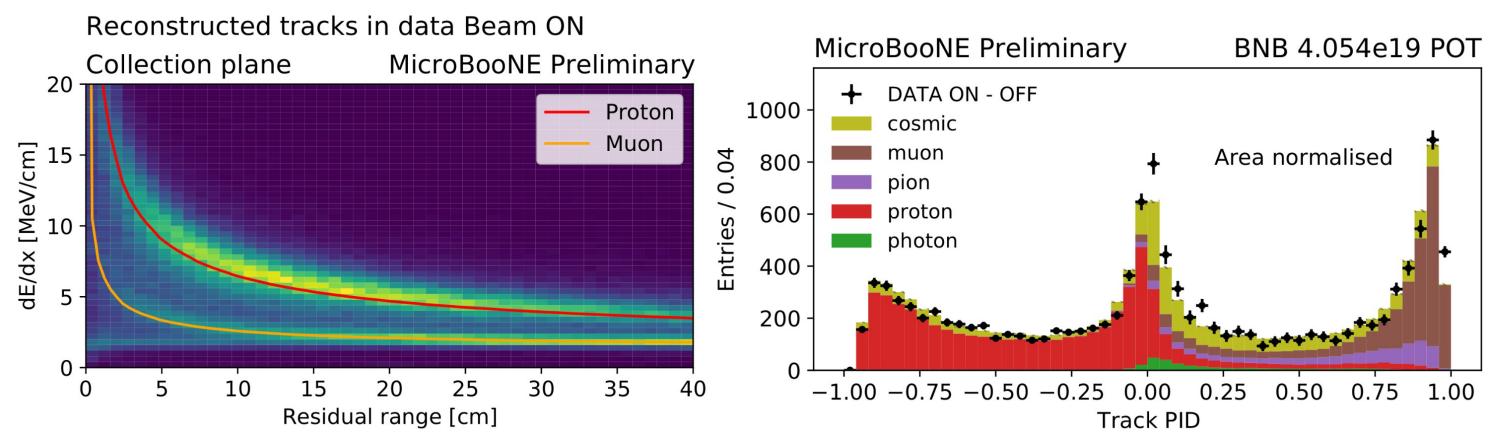

Figure 2: (Left) Histogram of the ionisation in function of the residual range for tracks in data. The red and yellow lines illustrate the predicted values using the Bethe-Bloch formula. (Right) Data to simulation comparison of the likelihood-ratio-based classification metric.

\subsubsection{Electron-Photon Separation}

An example of a data event containing a high-energetic electron shower candidate is given in the right panel of fig. 3. Separation of electron and photon showers is important both to understand the nature of a possible low-energy excess and discrimination against neutral current interactions with a $\pi^{0}$ in the final state. A boosted decision tree (BDT) was trained using simulated events, the separation power is shown in the right panel of fig. 3 .

\section{Charged-Current Electron Neutrino Selection}

After the cosmic rejection described in section 2, and an electromagnetic shower requirement, the $v_{e} \mathrm{CC}$ purity is $O(5 \%)$. The main backgrounds are shown in the left panel of fig. 4. The final $v_{e} \mathrm{CC}$ selection combined the particle identification results of section 3 in a boosted decision tree to achieve a $v_{e}$ CC purity above $50 \%$. The middle and left panel of fig. 4 demonstrate the inclusiveness 

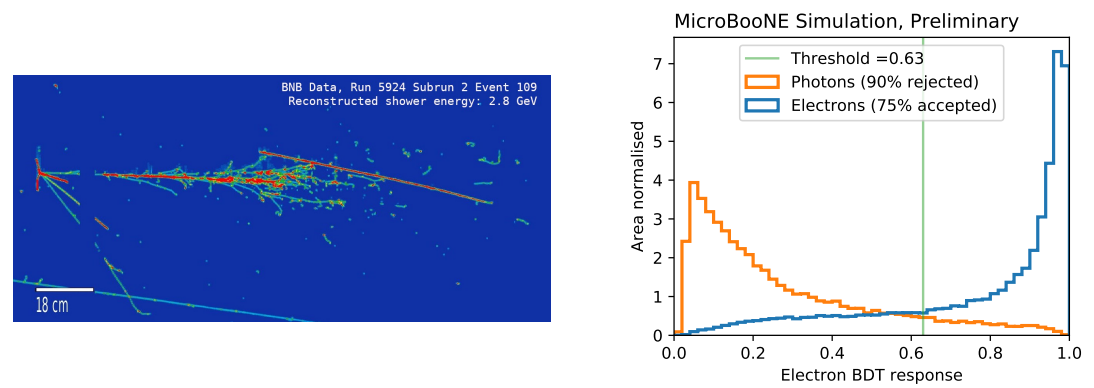

Figure 3: (Left) $v_{e}$ CC candidate event to illustrate an example of a high-energetic electromagnetic shower. (Right) $e-\gamma$ separation performance obtained by the electron classifier on simulation. The BDT electron score is shown for true electrons (blue histogram) and for background photons (orange histogram). The green line indicates the score at which $90 \%$ of the background photons are rejected.
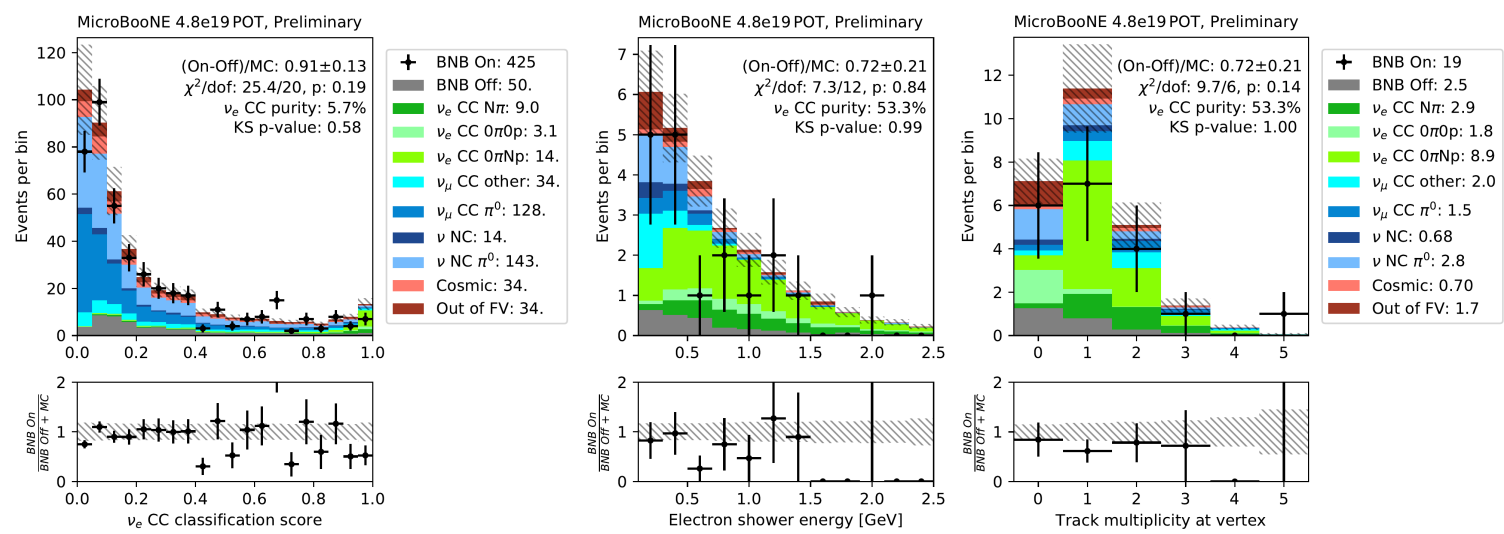

Figure 4: (Left) BDT response of the $v_{e} \mathrm{CC}$ inclusive event classifier. (middle) Reconstructed electron shower energy distribution after the selection. (right) Track multiplicity near the vertex for selected $v_{e}$ CC candidates.

of the selection; the selected events cover a wide range in both shower energy and vertex multiplicity. The selection efficiency integrated over the neutrino energy and different topologies is $18 \%$.

Figure 5 shows the resolution of the electron kinematics of selected events in simulation. The electron energy is reconstructed within approximately $20 \%$. The resolution of the angle between the neutrino beam and the outgoing electron is reconstructed with a $3^{\circ}$ resolution.

\section{High-Energy Sideband \& Outlook}

As mentioned in the introduction, low-energy events with electromagnetic showers are largely blinded while developing analyses. To understand low-energy shower formation in liquid argon, charged-current $v_{\mu}$ events with neutral pions in the final state are used [8, 9]. To understand electron neutrino interactions in liquid argon, two pathways are pursued simultaneously. First, $v_{e}$ events coming from the NuMI neutrino beam that send neutrinos off axis into the MicroBooNE LArTPC are studied [10]. Second, high-energy events, with a reconstructed energy above $1.05 \mathrm{GeV}$, from the Booster Neutrino Beam are studied with high statistics. The latter approach, shown here, has 

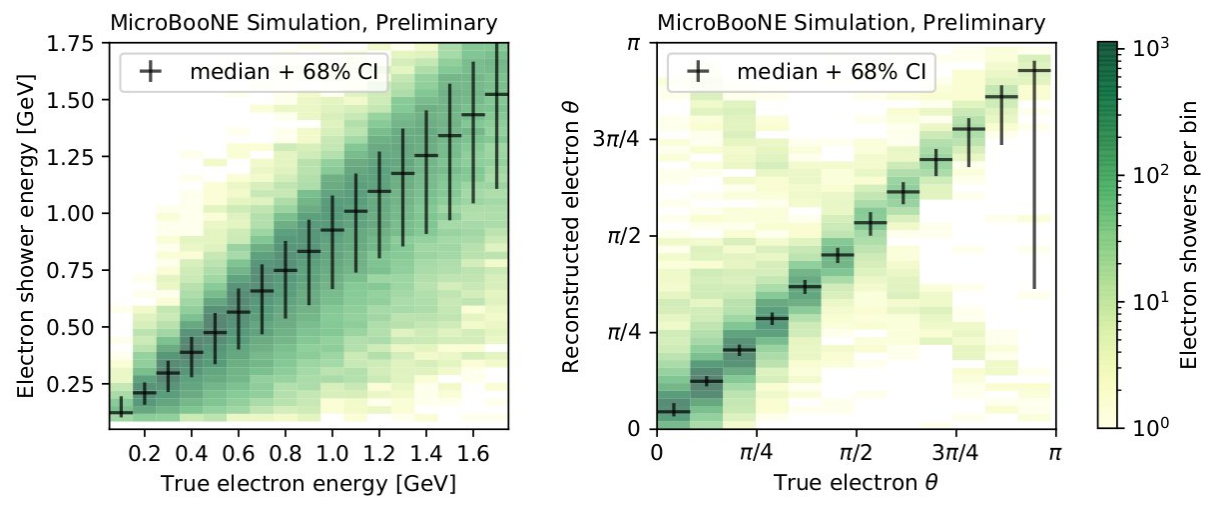

Figure 5: Resolution for the electron variables after the $v_{e} \mathrm{CC}$ selection. The colour scale is logarithmic. In black, the median and $68 \%$ confidence interval are given, binned in the true electron kinematics.

the benefit of being similar to the low-energy events in both beam flux properties and angular distributions.

Figure 6 shows the ionisation at the start of the shower and the shower vertex distance, bot variables that enable electron photon separation and are used as input of the shower classification introduced in section 3 .
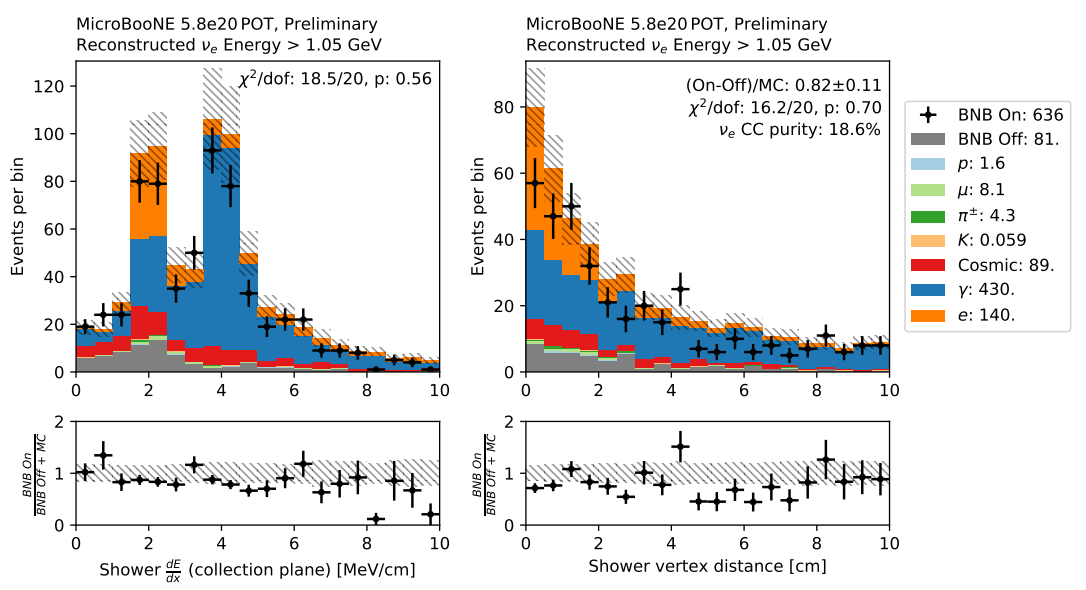

Figure 6: Reconstructed shower ionisation at the start of the shower (left) and shower distance from the reconstructed interaction vertex (right). Only events with a reconstructed energy above $1.05 \mathrm{GeV}$ are included. The simulation is divided by particle type and the data corresponds to $5.8 \times 10^{20}$ protons on target.

The kinematic properties of the electron shower of selected $v_{e} \mathrm{CC}$ candidates are given in fig. 7. At these higher energies, an expected purity of $O(80 \%)$ is reached.

The full details of this selection, together with analyses aimed at specific topologies that dominate the low-energy electron interactions can be found at [11].

\section{References}

[1] P.A. Machado, O. Palamara and D.W. Schmitz, The short-baseline neutrino program at Fermilab, Annual Review of Nuclear and Particle Science 69 (2019) 363. 

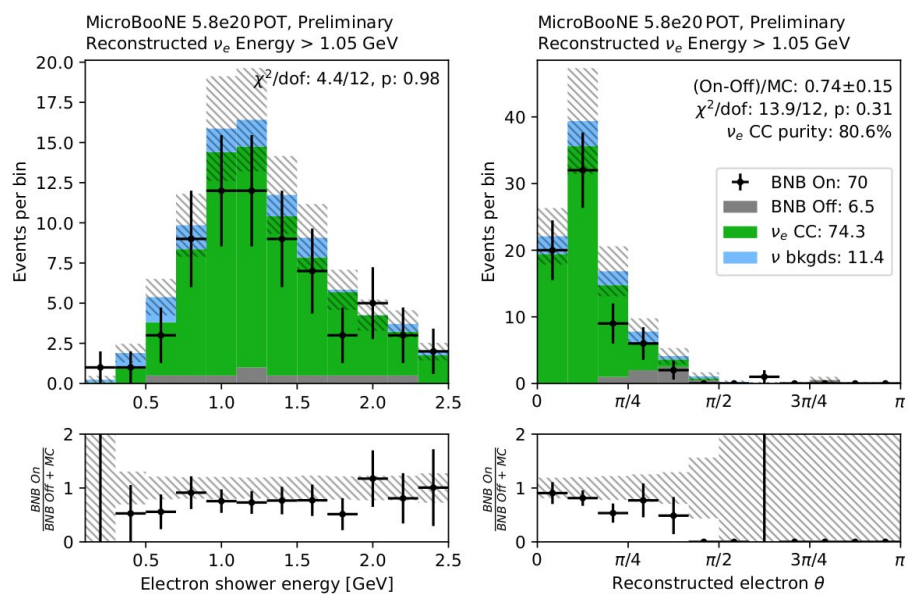

Figure 7: Reconstructed electron candidate kinematics after the selection. Only events with a reconstructed energy above $1.05 \mathrm{GeV}$ are included. The data shown corresponds to $5.8 \times 10^{20}$ protons on target.

[2] MiniBooNE collaboration, The Neutrino Flux prediction at MiniBooNE, Phys. Rev. D 79 (2009) 072002 [0806. 1449].

[3] MiniBooNE collaboration, Significant excess of electron-like events in the MiniBooNE short-baseline neutrino experiment, Phys. Rev. Lett. 121 (2018) 221801 [1805. 12028].

[4] M. Collaboration, Ionization electron signal processing in single phase lartpcs. part $i$. algorithm description and quantitative evaluation with MicroBooNE simulation, JINST 13 (2018) P07006-P07006 [1802 . 08709].

[5] MicroBooNE collaboration, Ionization electron signal processing in single phase LArTPCs. Part II. Data/simulation comparison and performance in MicroBooNE, JINST 13 (2018) P07007 [1804 . 02583].

[6] MicroBooNE collaboration, The pandora multi-algorithm approach to automated pattern recognition of cosmic-ray muon and neutrino events in the MicroBooNE detector, Eur. Phys. J. C (2018) 1 [1708.03135].

[7] MicroBooNE collaboration, Cosmic ray background rejection with wire-cell lartpc event reconstruction in MicroBooNE, MICROBOONE-NOTE-1084-PUB (2020) .

[8] MicroBooNE collaboration, Reconstruction and Measurement of $O(100) \mathrm{MeV}$ Energy Electromagnetic Activity from $\pi^{0} \rightarrow \gamma \gamma$ Decays in the MicroBooNE LArTPC, JINST 15 (2020) P02007 [1910.02166].

[9] MicroBooNE collaboration, First measurement of $v_{\mu}$ charged-current $\pi^{0}$ production on argon with the MicroBooNE detector, Phys. Rev. D 99 (2019) 091102 [1811. 02700].

[10] MicroBooNE collaboration, Automated selection of electron neutrinos from the numi beam in the MicroBooNE detector and prospects for a measurement of the charged-current inclusive cross section, MICROBOONE-NOTE-1054-PUB (2018) .

[11] MicroBooNE collaboration, Search for electron neutrinos in multiple topologies with the MicroBooNE experiment, MICROBOONE-NOTE-1085-PUB (2020) . 